\title{
Novel Ideas For Thermal Management Of Filament LED Light Bulbs
}

\author{
https://doi.org/10.3991/ijoe.v17i08.23695 \\ Mika Maaspuro \\ Aalto University, Finland \\ mika.maaspurodaalto.fi
}

\begin{abstract}
The thermal conditions of a filament LED retro light bulb has been investigated. In such light bulbs, LEDs are mounted on filaments which have glass or sapphire substrate and are surrounded by yttrium-aluminium-garnet (YAG). Heat management is challenging as a heatsink cannot normally be used. Instead, heat removal is enabled by using helium as the filling gas. As this provides less efficient heat removal than using a heatsink attached directly to an LED module, LEDs operate in filament LED bulbs typically at rather high temperatures. In this paper thermal performance of a typical filament LED bulb has been studied. LED temperatures and gas flows inside the bulb has been resolved for cases of different orientations of the bulb. Thermal conditions for different filling gases have been resolved. All this has been done using finite element method (FEM) simulations. The main target of this study has been to find out novel solutions for thermal management of filament LED bulbs. Two heat removal solutions have been studied, namely the use of an internal heatsink and an internal piezoelectric oscillating cantilever fan. The results show that solutions can indeed reduce temperature of LED chips and increase the lifespan of the LED bulb.
\end{abstract}

Keywords - filament LED, retro LED light bulb, thermal management of an LED bulb

\section{Introduction}

In the market of LED luminaires retro type solutions have still large market share. Customers favour traditional lamp bulbs, but are aware about the benefits LED technology can provide. Therefore, LED light bulbs are still sold in large quantities. Manufacturers are responding this request by offering new ideas of LED light bulbs. The mainstream is filament LED bulbs. Their outlook resembles as closely as possible the traditional incandescent light bulb.

The first filament LED string bulbs used external heatsink, but heat removal wasn't equally efficient as with LEDs mounted on a metal core printed circuit board (MCPCB). An improved thermal solution uses suitable filling gas to remove heat from the LEDs to the surface of the bulb. In this case the bulb no longer can be made of plastics, but is made of glass to ensure gastight. For an efficient heat remove the substrate of LEDs should be a material having a high heat transfer coefficient. Suitable materials are glass, 
sapphire, transparent ceramic or perforated copper. In a retro type LED bulb the driver is mounted inside the screw base of the lamp. In the most common types bases for retro light bulbs are E27 and E14, both of them can easily encapsulate an LED driver. The most common driver type is non-isolating Buck converter. It can be easily mounted inside the base. Attention has to be paid to the heat remove from the driver as well. This paper presents novel solutions for thermal management of filament LED bulbs. The paper is arranged as follows: Chapter 2 presents conventional heat management methods and serves as a short review on previous studies. Chapter 3 presents the particular LED bulb which was investigated. Chapter 4 introduces the underlying physics. Chapter 5 presents the created Multiphysics model. Chapter 6 presents simulation results for the filament LED bulb. Chapter 7 presents two novel ideas for heat management and simulation results for them. Finally, conclusions are presented in chapter 8 .

\section{Thermal management of filament LED bulbs}

These kind of LED lamps use filling gas for conducting dissipation heat out of the bulb and to keep the operation temperature of the LEDs at safe level. Steady-state simulations with various filling gases have been executed. Simulation results clearly show that heat is conducted through the filling gas to the glass bulb and convected to air from the outer surface of the bulb. Other heat conduction routes are less significant. The properties of the filling gas are the most important. There are just few pure gases which can be considered for the purpose. Substrate material of the LEDs shows also importance. The formation of YAG material affects on the heat dissipation.

This paragraph serves a review for various methods to thermal management of filament LED bulbs. The filling gas conducts heat from the filaments to the bulb and the base. Helium due to the small molecular size is the best among the gases which could be considered. It will be shown later by simulations that air or its main components like nitrogen have much too low thermal conducting coefficient to be considered to this purpose.

Helium is the filling gas used in filament LED bulbs. Helium $(0.144 \mathrm{~W} / \mathrm{m} \cdot \mathrm{K})$ is an expensive gas, but there are no really alternatives for it. Hydrogen $(0.163 \mathrm{~W} / \mathrm{m} \cdot \mathrm{K})$ would be better thermal conductor, but for other reasons not a suitable filling gas. [1] studied the possibility to reduce the ratio of helium gas while still maintaining sufficient heat dissipation characteristics. They found that the ratio of helium gas can be reduced to $80 \%$ when both luminous flux and color temperature still remains in a typical level. By that way cost reduction in the production will be achieved at the same time nominal luminous flux will be maintained. Adding nanoparticles to the filling gas can improve thermal conductivity. [2] introduces $\mathrm{Al}_{2} \mathrm{O}_{3}$ with helium as a suitable nanofluid.

Not only the thermal characteristics of the filling gas, but also size and thickness of the bulb and orientation of the filament LEDs, phosphor layer size and its form must be considered. Thermal and optical characteristics of the light bulb have to be considered together. In the LED filaments, LED junction and chip temperatures can be reduced by increasing the number of chips. By that way the electrical power dissipation will be distributed to many components. 
Some light bulbs use single spring-like flexible LED filament structure. The spring can be mounted vertically or horizontally compared to the orientation of the bulb. Authors of [3] have studied spring-like LED filament bulbs using simulations and experiments. They find that LED chip temperature is related to the pitch and pending radius of the filament. The temperature increases as the inclination angle improves. Considering also the optical characteristics an optimal shape of the LED filament can be solved.

[4] also studied spiral-like LED filaments and different filament arrangements in light bulbs. They found that vertical arrangement is better than horizontal one when considering both power efficiency and luminous flux. In vertical arrangement the filling gas flow speed is higher than in horizontal one. The filament arrangement, bulb orientation and stretch height of the filament effect on gas flows. While studying different filament arrangements the maximum power efficiency and luminous flux was reached simultaneously at the stretch height of $5 \mathrm{~cm}$. The results based on measurements and simulations. Difference with measurements and simulations was around $1.1 \%$. They concluded that the vertical filament arrangement has the best thermal and illuminance performance.

Thermal dissipation enhancement of LED filament bulb by ionic wind has been studied by [5]. Ionic wind can be created by applying a high voltage over the gas volume of the bulb. This can be done using needle electrodes. An ionic wind blower is created by applying a high $\mathrm{DC}$ voltage, typical at $\mathrm{kV}$ level between the electrodes. This causes charged electrons to start drifting between the electrodes which in turn generates an air flow. The benefits of ionic wind blower compared to a fan are non-moving components, fast response time, noiselessness, and low maintenance costs. In their experiment with helium filled LED bulb, the filament surface temperature was measured to be $130{ }^{\circ} \mathrm{C}$ and with $9 \mathrm{kV}$ ionic wind blower the temperature reduced approximately by $15^{\circ} \mathrm{C}$.

Finite Element Method (FEM) simulation provides an easy method for finding internal temperature of a lighting bulb without breaking the bulb for assembling a temperature sensor inside. [6] presents simulation results for a traditional incandescent light bulb.

\section{The simulated LED bulb}

Multiple filament LED bulbs were dismantled and examined. Some of the studied LED bulbs had no thermally enhanced material (TEM) filling in the lamp base. In those cases the main components of the driver are thermally poorly connected. Specially the surface temperature of the controller circuit keeps relatively high. Measurements verify the high surface temperature which was clearly observed in the simulations. The benefits of thermal silicon filling in the base are evident and becomes more significant in the proposed thermal improvement. 


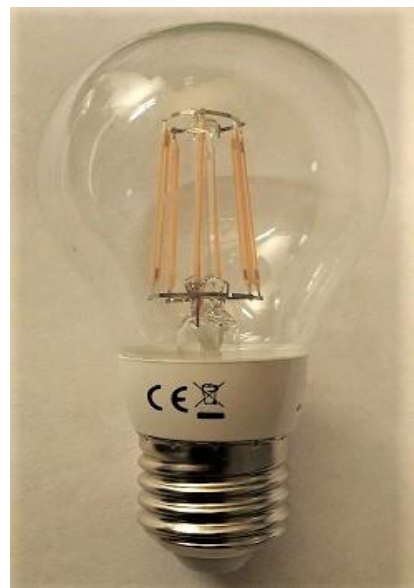

Fig. 1. The investigated $5 \mathrm{~W}$ filament LED bulb.

\section{The underlaying physics}

Heat transfer in solids and fluids as well fluid dynamics are involved. The heat transfer process is non-isothermal and the filling gas is modelled as slightly compressible gas. Gas fluids are considered to be single phase fluids and continuum. In these conditions the following fundamental laws [7] are coverning the physical problem:

- conservation of mass

- conservation of linear momentum

- conservation of energy

The first law can be written in the form

$$
\frac{\partial \rho}{\partial t}+\nabla \cdot(\rho \boldsymbol{u})=0
$$

$\rho$ is density, $\mathbf{u}$ is velocity vector

The second law can be written in many forms. In fluid mechanics it is also known as Navier-Stokes equation.

$$
\left.\rho\left[\frac{\partial \boldsymbol{u}}{\partial t}+(\boldsymbol{u} \cdot \nabla) \boldsymbol{u}\right)\right]=\nabla[-p \boldsymbol{I}+\boldsymbol{K}]+\boldsymbol{F}
$$

$\mathrm{p}$ is pressure, $\mathbf{K}$ is the viscous stress tensor and $\mathbf{F}$ is volume force vector, often caused by gravity. The third equation can also be written in many different ways,

$$
\rho C_{p}\left[\frac{\partial T}{\partial t}+(\boldsymbol{u} \cdot \nabla) T\right]=-(\nabla \cdot \boldsymbol{q})+\boldsymbol{K}: \boldsymbol{S}-\frac{T}{\rho} \frac{\partial \rho}{\partial T}\left(\frac{\partial p}{\partial t}+(\boldsymbol{u} \cdot \nabla) p+\boldsymbol{Q}\right.
$$

where $\mathrm{Cp}$ is the specific heat capacity, $\mathbf{q}$ heat flux vector, $\rho$ is density, $\mathbf{K}$ is the viscous stress tensor, $\mathbf{S}$ is the strain-rate tensor, $\mathrm{p}$ is pressure and $\mathbf{Q}$ contains all heat sources. 


\section{$5 \quad$ The simulation model}

The simulation was done by using Comsol Multiphysics software versions 5.5 and 5.6 with its heat transfer and CFD modules. 3D model was created by using the CAD tool of the software. To speed up the simulation symmetry of the bulb was used. It is possible to restrict the model to one fourth by using 2-axis symmetry. The driver shows no 2-axis symmetry, but to shake of simplicity, the main components on the driver PCB can be slightly shifted towards the centerline to establish 2-axis symmetry. This does not cause a significant error from thermal point of view.

The filament LED string is modelled with thermally well conducting layer and LEDs as boundary heat layers of zero thickness. Above the LEDs and substrate is the rather thick YAG layer. Substrate is connected to aluminium leads which are the electrical connectors to the LEDs. The LED bulb under investigation has six filament LED strings mounted on the "Christmas tree" constellation. The electrical wires are made of steel and leads to the driver and the base live connector. The bulb is made of glass. Filling gas is defined as a fluid.

LED chips have dimensions of $300 \mu \mathrm{m} \times 300 \mu \mathrm{m}$ and thickness of $100 \mu \mathrm{m}$. Each LED filament has 28 LED chips. LED chips are modelled as domain heat sources. The controller circuit of the driver which includes a switching MOSFET and an inductor. The actual power levels are set according to the electrical measurements of the driver.

Ambient temperature is set to $23^{\circ} \mathrm{C}$. Atmospheric pressure to $1 \mathrm{~atm}$. External natural heat convection conditions are established. The software provides automatic methods to adjust the convection coefficients which takes into account the LED bulb orientation (up/down). Simulation solves the stationary case.

\subsection{Meshing}

The quarter model has approximately 663000 elements. Average element quality (skewness) is 0.6673 and minimum quality is 0.0088 . Swept meshing has been used in parts where heat transfer is largely one directional, tetrahedral elements are used elsewhere. Mesh density various between different parts of the LED bulb. Boundary meshing is required for the CFD simulation.

\subsection{Simulations and solvers}

Stationary solution for heat transfer and CFD will be solved in case they are fully coupled, meaning the heat affects to the fluid conditions and fluids to the thermal conditions. Typically for this kind of combined simulation numerical difficulties arise. One common method is to step the dynamic viscosity of the gas. This can be done by using a setting stepping variable in material parameters of the gas. By stepping the dynamic viscosity for example like: $1000 \mathrm{x}, 100 \mathrm{x}, 10 \mathrm{x}$, finally $1 \mathrm{x}$ and using previous solution as the initial solution to the next one, the right solution will be reached. This method was found necessary also in these simulations.

Segregated solver method was used. Compared to the direct method, segregated method conserves memory usage. Multiphysics selects the optimal solver which in this 
case is GMRES (the Generalized Minimal Residual method). GMRES is a widely used iterative method for the numerical solution of a nonsymmetric system of linear equation. In general, GMRES provides accurate solutions. Transfer from v. 5.5 to v. 5.6 numerical problems came out. Changing the solver to FGMRES (the Flexible GMRES) avoided these problems and even reduced the computing time, but solutions shown some divergence to the previous ones. Returning to use GMRES and by manually adjusting the solver parameters in v. 5.6 both for fluid flow as well as heat transfer variables (set to non-linear based error norm) the difficulties were finally avoided and previously found solutions were restored.

\subsection{Computing hardware}

Simulations were executed using a computing cluster which provides sockets composed of Intel Xeon Gold 6248 or E5-2680 CPUs running at $2.5 \mathrm{GHz}$. Simulations were executed using a computing cluster which provides multiple sockets each having large number of efficient CPUs. For these simulations the cluster provides up to 40 cores and up to 200 GB memory. The used cluster may not provide much higher speed compared to the best PC's, but it allows to run multiple simulations parallel with a huge available memory. Simulation time for heat transfer only, without CFD was just about 2 minutes and complete Multiphysics simulation with coupled heat transfer and CFD between 5-12 hours.

\section{Simulation results of the filament LED bulb}

Table 1 shows simulation results for various filling gases. The table lists the average, maximum and minimum temperatures of the LED chips. It clearly shows that only hydrogen and helium have thermal conductivity high enough. For other reasons, only helium can be considered.

Figure 2 shows simulated surface temperature of the bulb. Table 2 compares the simulated and measured surface temperatures. Gravity direction is downwards. For that reason, the hottest surface point is at the top of the bulb. The simulated and measured values match well. The biggest difference is in the temperature of the bulb top.

Table 1. Simulated LED chip temperatures for various filling gases.

\begin{tabular}{|l|c|c|c|}
\hline \multicolumn{1}{|c|}{ Gas } & $\begin{array}{c}\mathbf{T}(\mathbf{a v g}) \\
{\left[{ }^{\circ} \mathbf{C}\right]}\end{array}$ & $\begin{array}{c}\mathbf{T}(\mathbf{m a x}) \\
{\left[{ }^{\circ} \mathbf{C}\right]}\end{array}$ & $\begin{array}{c}\mathbf{T}(\mathbf{m i n}) \\
{\left[{ }^{\circ} \mathbf{C}\right]}\end{array}$ \\
\hline Hydrogen & 85.23 & 90.43 & 70.90 \\
\hline Helium & 95.30 & 100.94 & 79.48 \\
\hline Neon & 133.19 & 141.17 & 110.35 \\
\hline Air & 153.82 & 162.20 & 128.66 \\
\hline Nitrogen & 154.49 & 162.89 & 129.23 \\
\hline Argon & 215.70 & 227.42 & 181.93 \\
\hline
\end{tabular}


Table 2. Simulated and measured bulb surface temperatures.

\begin{tabular}{|l|c|c|}
\hline & Simulated $\left[{ }^{\circ} \mathbf{C}\right]$ & Measured $\left[{ }^{\circ} \mathbf{C}\right]$ \\
\hline Bulb top temp. & 46.7 & 44.0 \\
\hline at $0.75 \times$ height & 41.8 & 41.6 \\
\hline at $0.50 \times$ height & 37.0 & 36.6 \\
\hline at $0.25 \times$ height & 37.6 & 37.9 \\
\hline
\end{tabular}

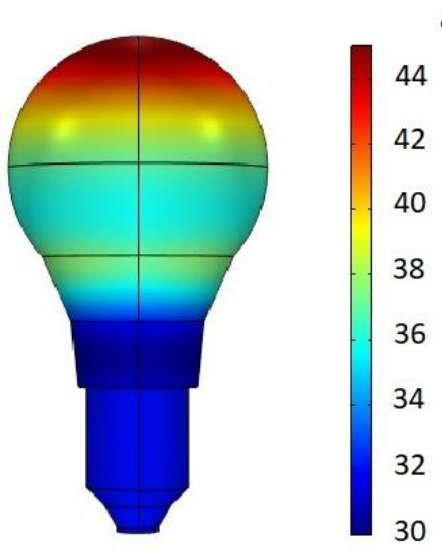

${ }^{\circ} \mathrm{C}$

44

42

40

38

36

34

32

30

Fig. 2. Simulated surface temperature.

Figure 3 and 4 shows the internal temperature of the bulb. Ambient temperature is $23{ }^{\circ} \mathrm{C}$. The highest temperature is found in LED chips which are closest the top. LED driver MOSFET in the base shows slightly elevated temperature. Figure 5 shows the internal gas flows, which appear as vortexes. Near the LED filaments gas flows upwards and near the surface of the bulb downwards. The maximum flow speed is close to $6 \mathrm{~cm} / \mathrm{s}$. Simulations were executed for several orientations of the LED bulb. Figure 6 shows the orientations and the gas flows. For tilt angles from 90 to 180 the results are closely similar.
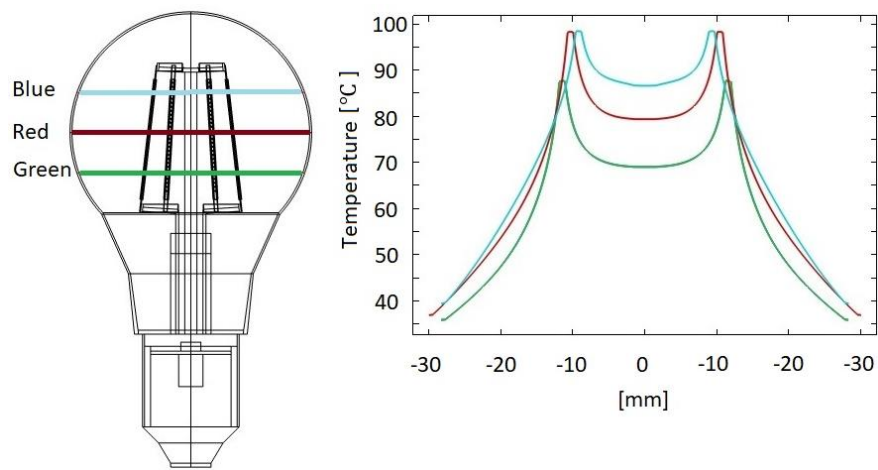

Fig. 3. Internal temperature of the bulb along horizontal lines. Gravity direction is downwards. 


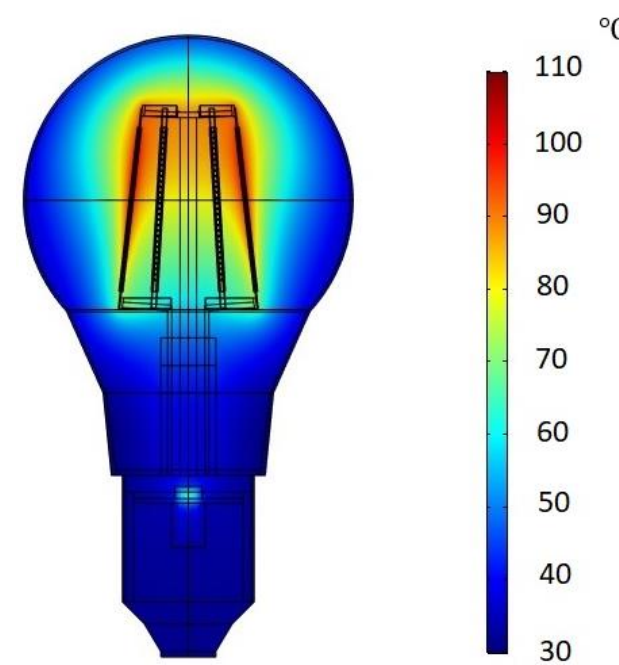

Fig. 4. Simulated temperature. The slice is cut at the center of the bulb.

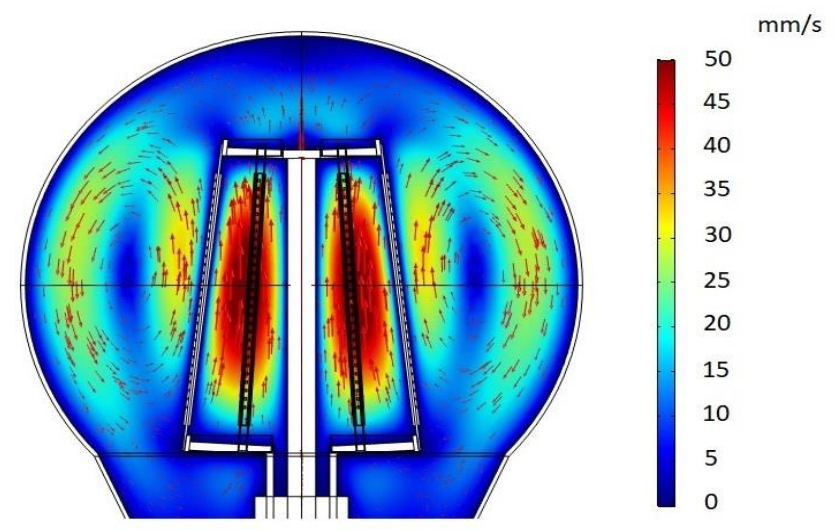

Fig. 5. Simulated gas flows in the bulb.

Table 3. Simulated temperatures of LED chips and gas flow speed in helium filled LED bulb in various geometrical orientations.

\begin{tabular}{|c|c|c|c|c|c|c|}
\hline Angle & & $\begin{array}{c}0 \\
\text { [deg.] }\end{array}$ & $\begin{array}{c}45 \\
\text { [deg.] }\end{array}$ & $\begin{array}{c}90 \\
\text { [deg.] }\end{array}$ & $\begin{array}{c}135 \\
\text { [deg.] }\end{array}$ & $\begin{array}{c}180 \\
\text { [deg.] }\end{array}$ \\
\hline $\begin{array}{l}\text { Temp. of the } \\
\text { LEDs }\left[{ }^{\circ} \mathrm{C}\right]\end{array}$ & $\begin{array}{l}\text { avg } \\
\max \\
\min \end{array}$ & $\begin{array}{c}95.30 \\
100.94 \\
79.48\end{array}$ & $\begin{array}{c}95.63 \\
101.69 \\
81.40\end{array}$ & $\begin{array}{c}96.08 \\
102.42 \\
85.57\end{array}$ & $\begin{array}{c}96.20 \\
100.76 \\
86.79\end{array}$ & $\begin{array}{l}95.08 \\
99.09 \\
86.26\end{array}$ \\
\hline Flow speed & avg & 1.75 & 1.57 & 1.23 & 1.38 & 1.75 \\
\hline$[\mathrm{cm} / \mathrm{s}]$ & $\max$ & 5.80 & 5.05 & 3.76 & 4.66 & 6.03 \\
\hline
\end{tabular}


$\mathrm{mm} / \mathrm{s}$

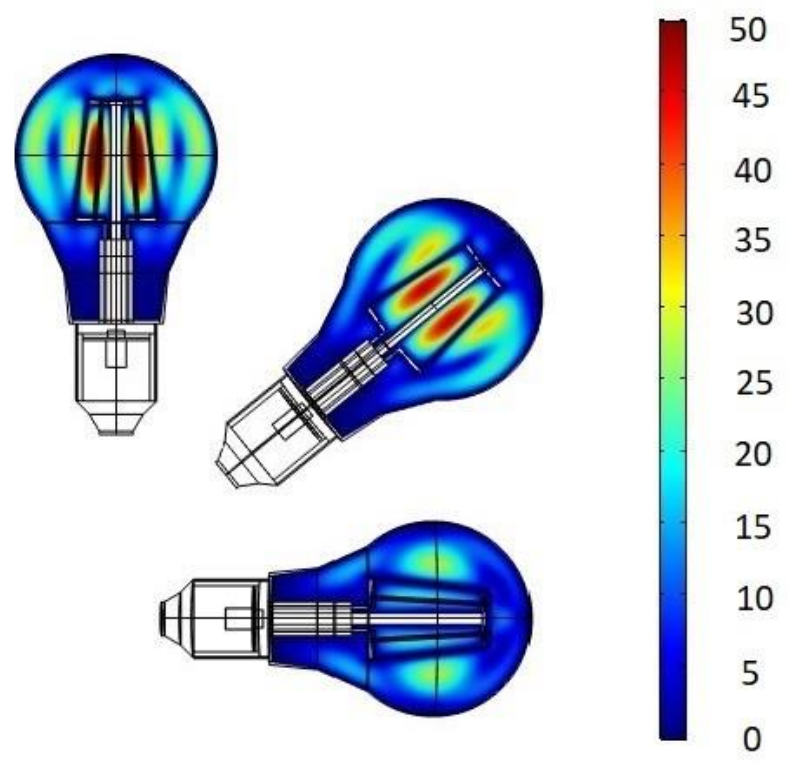

Fig. 6. Gas flows inside the bulb in various bulb orientations (inclined 0, 45 and 90 degrees).

Table 4. Simulated temperatures of LED chips and helium gas flow with different bulb thicknesses.

\begin{tabular}{|l|c|c|c|c|}
\hline $\begin{array}{c}\text { Bulb } \\
\text { thickness }\end{array}$ & & $\begin{array}{c}\mathbf{0 . 5} \\
{[\mathbf{m m}]}\end{array}$ & $\begin{array}{c}\mathbf{1 . 0} \\
{[\mathbf{m m}]}\end{array}$ & $\begin{array}{c}\mathbf{1 . 5} \\
{[\mathbf{m m}]}\end{array}$ \\
\hline LED's temp. $\left[{ }^{\circ} \mathrm{C}\right]$ & avg & 95.30 & 94.99 & 94.62 \\
& $\min$ & 100.94 & 100.51 & 100.04 \\
& $\max$ & 79.45 & 79.37 & 79.21 \\
Gas speed & & & & \\
{$[\mathrm{cm} / \mathrm{s}]$} & avg & 1.75 & 1.69 & 1.63 \\
& $\max$ & 5.80 & 5.62 & 5.45 \\
\hline
\end{tabular}

\section{$7 \quad$ Novel ideas for thermal management}

\section{Internal heatsink}

Use of a heatsink can improve heat conduction in a bulb. An internal heatsink can provide a heat path from filling gas to the base of the bulb. From the bulb heat conducts to a lighting device and further. The investigated light bulb was set to a typical lighting device and it was found that base temperature sets around $32{ }^{\circ} \mathrm{C}$ when ambient temperature is $23{ }^{\circ} \mathrm{C}$. All the simulations in this study use these boundary conditions. A copper heatsink was included in the simulation model. The heatsink has fins on the both ends in order to efficiently sink and source heat. The heatsink has a volume of $0.323 \mathrm{~cm}^{3}$ and 
weight of $2.9 \mathrm{~g}$. Simulations show that average temperature of LED chips reduced approximately by $20^{\circ} \mathrm{C}$. This can be seen in Figure 8 which scale is set to the same as in Figure 4 to make these figures easily comparable.

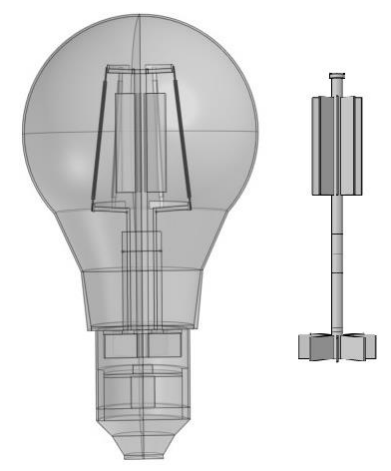

Fig. 7. The internal heatsink

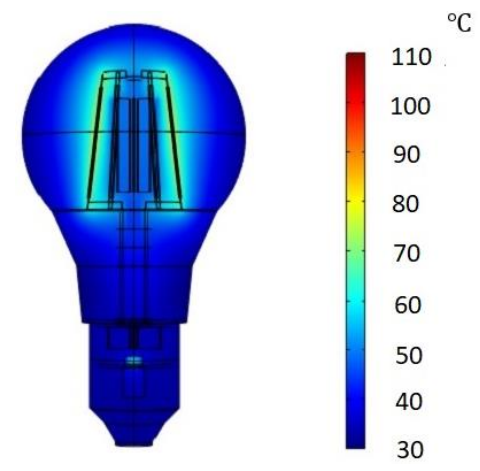

Fig. 8. Simulated temperatures of the filament LED bulb with the internal heatsink.

\section{Piezoelectric oscillating cantilever fan}

The simulation results (table 3.) show that the temperature of LEDs is lower when gas flow speed inside the bulb is higher. Stronger gas flows increase thermal conduction to inner surface of the bulb where heat is conducted through the glass and finally convected to the ambient. This leads to an idea to increase the gas flows by an internal fan.

Piezoelectric oscillating cantilever fan is built up with piezoelectric element and a flexible beam attached to it. When ac-voltage is applied over the element the beam starts oscillating and producing an air flow. The oscillation amplitude and flow volume will be maximized by setting the driving voltage frequency matching the mechanical resonance of the structure. The produced fluid flow is the highest at zero deflection of the beam. The minimum is reached at the beam maximum amplitude [9]. A piezoelectric oscillating cantilever fan is a reliable, highly energy efficient, silent, reliable, simple constructed, inexpensive and has a long lifetime [10]. Typically, it requires voltage of 60-230 VAC and consumes just few tens of milliwatts of power. By adjusting beam dimensions, the resonance can be set to match the line voltage frequency. By this way the fan requires no additional electrical driver for operation. In case the resonance differs from the line frequency, an additional driver for the fan is needed, but the driver can be mounted inside the bulb base.

[8] has studied and characterized the performance of piezoelectric oscillating cantilever fan, its operating parameters and the effects of the proximity of enclosure walls. They studied both Mylar and steel beams and found that both of them can operate approximately equally efficiently, but having small difference in amplitudes and flow speeds. However, the mylar blade seems to be slightly better (20\%). The fans they studied produced amplitude A of max 6.5 (steel) $-10 \mathrm{~mm}$ (Mylar) with frequency of $60 \mathrm{~Hz}$ (Mylar) or $113 \mathrm{~Hz}$ (steel). The maximum flow rate conditions yield nearly $30 \mathrm{l} / \mathrm{min}$, 
while the greatest static pressure generated was only $6 \mathrm{~Pa}$. Power consumption was in range $10-45 \mathrm{~mW}$.

In this study a commercial piezo-fan and its characteristics as well as equal fan with steel beam were used on the basis for these simulations. The fan with Mylar beam has piezo element of size $12 \mathrm{~mm}$ x $41 \mathrm{~mm}$ and beam of size $12.7 \mathrm{~mm}$ (width) x $36 \mathrm{~mm}$ (exposed length). The fan with steel beam has beam size of $12.7 \mathrm{~mm}$ (width) x $23 \mathrm{~mm}$ (exposed length). Resonance frequency is $50 / 60 \mathrm{~Hz}$ (Mylar), $113 \mathrm{~Hz}$ (steel). The fan with Mylar beam is too large to be set in the studied LED lamp, inside A60 bulb with E27 base. Therefore, simulations have been made for the fan is with steel beam. This choice is also reasonable when considering the operating temperature inside the bulb. If the filament LEDs had been constructed differently, it could be possible to use a larger fan.

$$
\frac{P}{P_{0}}=1-\left(\frac{Q}{Q_{0}}\right)^{q}
$$

where $\mathrm{P}$ is the pressure caused by the flow rate $\mathrm{Q} . \mathrm{P} 0$ is the zero pressure and $\mathrm{Q} 0$ is the equivalent flow rate. According to [8] $\mathrm{q}$ is approximately 1.6 for both fans with Mylar or steel beams. Fans with Mylar beams oscillate with higher amplitudes than fans with steel beams. On the other hand, fans with steel beams generate a higher pressure. As the experimental results [8] have been made for a fan in air, they cannot directly been converted to the case the gas is helium. The density of helium is a $1 / 6$ of air. Therefore, a fan driven in helium compared to air shows a higher amplitude but reduced pressure. In case of a flexible beam like Mylar the amplitude increases much, but in in case of steel less. Comparable parameters for a fan in helium has been calculated from the results for a fan with steel beam and max amplitude of $6 \mathrm{~mm}$. These values are pessimistic and in reality the fan may show a better performance.

The pressure $\mathrm{P}$ and flow speed the fan generates can be calculated by using the following equations:

$$
\begin{gathered}
P=P_{0} \rho f^{2} A D \\
\mathrm{Q}=Q_{0} \mathrm{fADL}
\end{gathered}
$$

where $\rho$ is density of the gas, A ist the amplitude and D ist the width and $\mathrm{L}$ is the length of the oscillating beam. Typically, a fan operates at frequency $f$ which is the first resonance mode of the structure. As the equation indicates pressure is dependent on gas density and strongly dependent on the frequency. Flow speed is less affected by the frequency. A fan which has a large amplitude typically operates at a lower frequency than a fan which features a lower amplitude. Amplitude decreases and frequency increases as length of a fan reduces.

Wall proximity effect becomes prominent with small enclosures. Nearby walls cause drag for the beam causing amplitude decrement or if the amplitude is attempted to maintain more energy must be supplied to the fan. The loss in amplitude may rise to tens of percent in case of a small enclosure. In general oscillating piezo fan features the best generated pressure and flow speed when it is operating without an enclosure. But in that case the generated air flow is the least directed. 

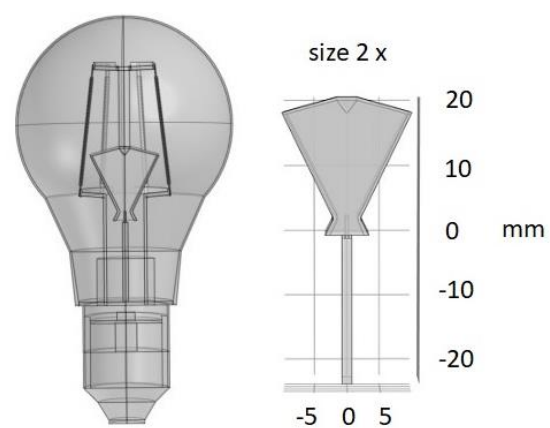

Fig. 9. Enhanced thermal management solution using internal piezo fan. Piezo fan enclosure on the right is shown with a double size.
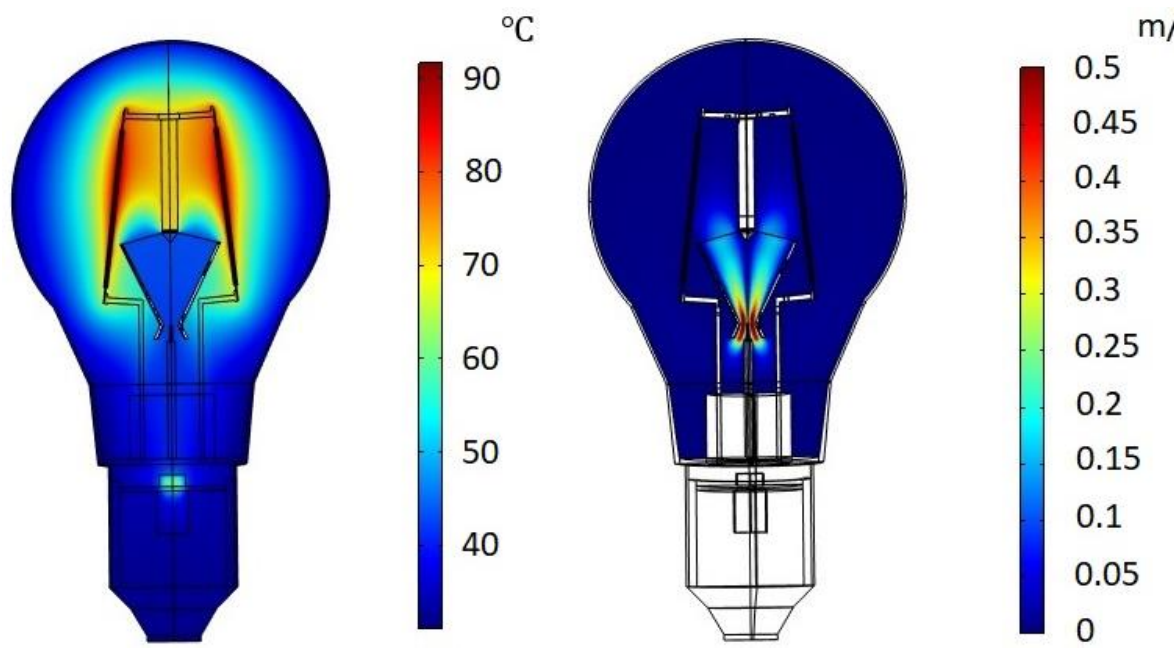

Fig. 10. Simulated temperature and gas flows of the bulb with an internal fan $(0.5 \mathrm{~Pa}, 10 \mathrm{l} / \mathrm{min})$.

Table 5. The basic and improved solutions - simulation results.

\begin{tabular}{|l|c|c|c|c|c|}
\hline & & Basic & Heat-sink & Fan 1 & Fan 2 \\
\hline Max pressure [Pa] & & & & 0.5 & 1 \\
Max flow [1/min] & & & & 10 & 10 \\
LED's temp. [ $\left.{ }^{\circ} \mathrm{C}\right]$ & awg & 95.30 & 75.43 & 86.39 & 85.21 \\
& $\max$ & 100.94 & 78.88 & 91.49 & 90.48 \\
& $\min$ & 79.48 & 64.73 & 72.98 & 71.92 \\
Hot LED's temp. $\left[{ }^{\circ} \mathrm{C}\right]$ & awg & & & 87.29 & 86.41 \\
Cool LED's temp. [ & & & 84.59 & 82.82 \\
& awg & & & & \\
Gas flow speed [cm/s] & & & & 0.79 & 1.61 \\
& awg & 1.75 & 1.05 & 135 & 247 \\
\hline
\end{tabular}




\section{Conclusions}

Thermal conditions of a typical 5W LED bulb were investigated. Helium has the second highest heat transfer coefficient among pure gases. In practice it is the only alternative for a filling gas in filament LED bulbs. Simulations showed that average temperature of LED chips is around $95-96{ }^{\circ} \mathrm{C}$ when the helium filled bulb is set in natural heat convection conditions. Hydrogen would provide a bit lower temperature, around $85^{\circ} \mathrm{C}$, but due to the apparent safety reasons, it cannot be used. Cases with four other gases were simulated, and in all cases the average LED ship temperature exceeded the maximum junction temperature, which for these kind of filament LED strings is typically around $125^{\circ} \mathrm{C}$. Simulations also shown that there are temperature differences among the LEDs. The difference is about $6{ }^{\circ} \mathrm{C}$, the highest temperatures are reached in the LEDs which locate nearest to the bulb top. Simulations showed the internal gas vortexes inside the bulb. Near the bulb surface the flow directs downwards and near the LEDs upwards. Orientation of the bulb effects on the gas flows and the average, minimum and maximum temperatures of the LED chips. There is also a relationship between the flow speeds and the temperatures. The lower the flow speeds the higher the temperatures. This leads to an idea to somehow increase the flow speeds for reducing the temperatures. Simulations shown the temperature profile inside the bulb. Naturally temperature is the lowest near the bulb surface. Simulations for a few different bulb glass thicknesses shown that increasing the thickness from 0.5 to $1.5 \mathrm{~mm}$ reduces slightly, less than $1{ }^{\circ} \mathrm{C}$ the average LED temperature.

Two ideas were considered for improving heat dissipation with filament LED bulbs. Internal heatsink could conduct heat from the filling gas to the base of the bulb, which normally has a lower temperature and provides a path for heat. With a copper made heatsink the average temperature of LED chips reduced to around $75^{\circ} \mathrm{C}$, featuring significant $20^{\circ} \mathrm{C}$ reduction. By adding something inside the bulb reduces the average gas flow speed. With the heatsink the average gas flow speed decreased from $1.75 \mathrm{~cm} / \mathrm{s}$ to $1.05 \mathrm{~cm} / \mathrm{s}$. The second idea was using a piezoelectric fan inside the bulb. Simulations with a typical piezoelectric fan with a metal beam and a thermally conducting envelope shown temperature reduction of approximately $10{ }^{\circ} \mathrm{C}$. Comparing temperatures of hot and cool LEDs indicates that temperature reduction due to the increased gas flow itself was around $3-4{ }^{\circ} \mathrm{C}$. With the fan the maximum gas flow speed is high, over $1 \mathrm{~m} / \mathrm{s}$. The size of A60 bulb sets severe limitations for the fan size. Also, the orientation of LED filaments poses difficulties for positioning the fan optimally. Obviously, the combination of a custom-made internal heatsink and an internal fan could provide the best improvement in temperature management.

\section{$9 \quad$ References}

[1] Chen, Y., Zeng, Q., Zhao, L., Li, Y., Huang, G. and Li, B. (2019). "Optimization of Helium Inflating on Heat Dissipation and Luminescence Properties of the A60 LED Filament Lamps", 5 pages, Hindawi International Journal of Photoenergy, Article ID 6292036. https://doi.org/10.1155/2019/6292036 
[2] Wang, J. C., Lin, C. Y. and Chen, T. C. (2013). "Thermal performance of a vapor chamberbased plate of high-power light emitting diodes filled with Al2O3 nanofluid", J. Nanosci. Nanotechno, vol. 13, no. 4, pp. 2871-2878. https://doi.org/10.1166/jnn.2013.7360

[3] Wang, L., Li, W., Xu, Y., Yang, B., Shi, M., Zou, J., Li, Y., Qian, X., Zheng, F., Yang, L. (2018). "Effect of different bending shapes on thermal properties of flexible light-emitting diode filament", 7 pages, Chinese Physics, B27, 110701. https://doi.org/10.1088/1674$1056 / 27 / 11 / 110701$

[4] Wang, W., Zou, J., Zheng, Q., Li, Y., Yang, B., Shi, M., Li, Y., Li, X., Zhang, C., Li, C., Chen, D. (2020). "The Effect of Different Filament Arrangements on Thermal and Optical Performances of LED Bulbs”, 10 pages, Applied Sciences, MDPI Journals, vol. 10, issue 4. https://doi.org/10.3390/app10041373

[5] Xu, C., Zhang, Z., Chu, J., Wu, J., Lei, X., Zheng, H., Liu, S. (2016). “Thermal dissipation enhancement of LED filament bulb by ionic wind", 17th International Conference on Electronic Packaging Technology, pp. 1212-1215. https://doi.org/10.1109/icept.2016.7583341

[6] Milanovic, I., Eppes, T. A., Russell, I. "Engineering Multiphysics Research", pp. 24-28, Vol. 7, No. 2, iJOE International Journal of Online and Biomedical Engineering. https://doi.org/10.3991/ijoe.v7i2.1641

[7] "CFD Module, User's Guide", Theory for the Single-Phase Flow Interfaces, pp. 149, Comsol Multiphysics, ver. 5.6. 2020.

[8] Kimber, M., Suzuki, K., Kitsunai, N., Seki, K., Garimella, S. V. (2009). "Pressure and Flow Rate Performance of Piezoelectric Fans", IEEE Transactions on components and packaging technologies, vol. 32, no. 4, pp. 766-775. https://doi.org/10.1109/tcapt.2008.2012169

[9] Jamalabadi, M. Y. A. "LBM simulation of piezo fan in square enclosure", International Journal of Numerical Methods for Heat \& Fluid Flow, Emerald Publishing Ltd, pp. 401426, vol. 30, no. 1. https://doi.org/10.1108/hff-05-2019-0409

[10] Maaspuro, M. (2016). "Piezoelectric oscillating cantilever fan for thermal Management of electronics and LEDs - A review", vol.63, pp.342-353, Microelectronics Reliability. https://doi.org/10.1016/j.microrel.2016.06.008

\section{Author}

Mika Maaspuro received the M.Sc. degree in electrical engineering (electronics) from Tampere University of Technology, Finland in 1989 and the Lic.Sc. degree (electronics) from Faculty of Mathematics and Natural Sciences in Turku University, Finland in 2010. He currently has a student position in the department of electrical engineering and automation in Aalto University, Finland.

Article submitted 2021-05-03. Resubmitted 2021-06-17. Final acceptance 2021-06-21. Final version published as submitted by the authors. 